\title{
MODEL DISCRIMINATION FOR REACTIONS WITH A STOP-EFFECT
}

\author{
JAN THULLIE \\ Polish Academy of Sciences, Institute of Chemical Engineering, 44-100 Gliwice, ul. Baltycka 5, Puland \\ and \\ ALBERT RENKEN
Ecole Polytechnique Fédérale, Institut de génie chimique, EPFL-Ecublens, CH-1015 Lausanne,
Switzerland
}

(Received 5 April 1993; accepted 27 May 1993)

\begin{abstract}
Several basic models presented in the literature for reactions with a stop-effect yield virtually the same results under steady state conditions. Such a situation takes place for dehydration of alcohols and the deamination of primary amines. It has been proved that a simple transient behaviour after a stop in the supply of the reactant will not make it possible to distinguish between the models. However, a considerable difference in the mean reaction rate under forced concentration oscillations between the models can be observed. The difference decreases with an increase in the cycling time.
\end{abstract}

\section{INTRODUCTION}

Parameter estimation and model discrimination are often the most important steps in the interpretation of experimental results, and immense progress has occurred in this field. Sometimes it is possible that a problem which is difficult to solve by standard methods can be solved by new techniques. Such a situation arises when investigating reactions with a stopeffect.

There are two basic models that explain this effect. One of them (I) assumes existence of two kinds of active sites taking part in the process; it was analysed by Nowobilski and Takoudis (1986). The second one (II) takes into account only one kind of active site and was used by Koubek et al. (1980a, b) to give an approximate description of his results. This model is well known as substrate inhibition in enzyme kinetics (Bailey and Ollis, 1977). Both models were analysed by Thullie and Renken (1991) under forced concentration oscillations (FCO), and it was concluded that discriminating between them under steady-state conditions is sometimes impossible.

It is important to note that sometimes the dynamic behaviour of the models after a stop in the supply of the reactant is very similar. However, the mean reaction rate under FCO can differ profoundly, and this gives a way to differentiate between them.

\section{ANALYSIS OF STEADY-STATE CONDITIONS}

Both models under consideration are based on the adsorption/desorption approach, and each model consists of several steps. It was confirmed by the experiments of Koubek et al. (1980a, b) that the most important steps are:

Model I:

$$
A+S_{1} \underset{k=1}{k_{1}} A S_{1}
$$

$$
\begin{gathered}
\mathrm{A}+\mathrm{S}_{2} \underset{k \cdot 2}{\stackrel{k_{2}}{\leftrightarrow}} \mathrm{AS}_{2} \\
\mathrm{AS}_{1}+\mathrm{S}_{2} \stackrel{\boldsymbol{k}_{3}}{\Rightarrow} \mathrm{C}+\mathrm{S}_{1}+\mathrm{S}_{2}
\end{gathered}
$$

Model II:

$$
\begin{gathered}
A+S \underset{k-1}{\stackrel{k_{1}}{\leftrightarrows}} A S \\
A S+A \underset{k-2}{\stackrel{k_{2}}{\leftrightarrows}} A S A \\
A S \stackrel{k_{3}}{\Rightarrow} C+S .
\end{gathered}
$$

The steady-state reaction rates are:

Model I:

$$
R_{s I}=\frac{k_{3} K_{1}[\mathrm{~A}]_{s}}{\left(K_{1}[\mathrm{~A}]_{s}+1\right)\left(K_{2}[\mathrm{~A}]_{s}+1\right)+k_{3} / k_{-1}}
$$

Model II:

$$
\begin{aligned}
R_{\text {SII }} & =\frac{k_{3} K_{1}[\mathrm{~A}]_{s}}{K_{1}[\mathrm{~A}]_{s}\left(K_{2}[\mathrm{~A}]_{s}+1\right)+\left(k_{3}+k_{-1}\right) / k_{-1}} \\
& =\frac{k_{3}[\mathrm{~A}]_{s}}{K_{m}+[\mathrm{A}]_{s}+K_{2}[\mathrm{~A}]_{S}^{2}}
\end{aligned}
$$

with $K_{m}=\left(k_{3}+k_{-1}\right) / k_{1}$ corresponding to the modified Michaelis-Menten constant for enzymatic reactions.

Three cases were investigated in which the same fit to differential reactor experiments were produced by

(1) several sets of kinetic parameters for one model,

(2) two models with different sets of kinetic parameters, and

(3) two models with the same set of kinetic parameters.

Let us analyse these three cases. The first one is quite frequently encountered in chemical kinetics, and 
has been the subject of several studies. For reactions with a stop-effect, the way to tackle is the same as for the second case and therefore it will not be discussed separately.

First, one should define what constraints upon kinetic parameters are given by a steady state for the second case. To make the investigation clearer we denote the second set of kinetic parameters by a prime ('), to differentiate them from the first set. The equality of the steady-state reaction rate gives

$$
\frac{R_{S I I}}{R_{51}}=\frac{k_{3}^{\prime} K_{1}^{\prime} H_{\mathrm{I}}}{k_{3} K_{1} H_{1 \mathrm{I}}^{\prime}}=1
$$

This relation will be helpful in analysing dynamic behaviour.

When parameters of both models are the same, we have the third case and

$$
\frac{R_{S I I}}{R_{S I}}=\frac{H_{I}}{H_{I I}}=1 .
$$

It is of interest that eqs (4) and (5) give these results only for a certain range of parameters. To determine this range, consider a ratio $\boldsymbol{R}_{\text {sII }} / \boldsymbol{R}_{\text {sI }}$ in general form by introducing eq. (4) and eq. (5) into it:

$$
\begin{aligned}
\frac{R_{\text {SII }}}{R_{\text {SI }}} & =1+\frac{K_{2}[\mathrm{~A}]_{s}}{K_{1}[\mathrm{~A}]_{s}\left(K_{2}[\mathrm{~A}]_{s}+1\right)+\left(k_{3}+k_{-1}\right) / k_{-1}} \\
& =1+\varphi .
\end{aligned}
$$

The function $\varphi$ can be defined as

$$
\varphi=\frac{K_{2}[\mathrm{~A}]_{s}}{K_{1}[\mathrm{~A}]_{\mathrm{s}}\left(K_{2}[\mathrm{~A}]_{s}+1\right)+1+k_{3} / k_{-1}} .
$$

It determines the discrepancy in the steady-state behaviour of both models. For a given set of kinetic parameters it depends on concentration $[\mathrm{A}]_{s}$.

The maximum value of $\varphi$ is obtained when

$$
[\mathrm{A}]_{s}=[\mathrm{A}]_{s o p}=\sqrt{\frac{1+k_{3} / k_{-1}}{K_{1} K_{2}}} \text { for } k_{1} \neq 0
$$

which is the value when both $\boldsymbol{R}_{S I}$ and $\boldsymbol{R}_{S I I}$ are at their maximum values. Introducing (10) into (9) results in

$$
\varphi_{\max }=\frac{1}{K_{1} / K_{2}+2 \sqrt{K_{1} / K_{2}\left(1+k_{3} / k_{-1}\right)}} .
$$

When $K_{1}$ tends to zero, [A] $]_{\text {sop }}$ tends to infinity. In this case $\varphi$ is determined by an asymptotic value

$$
\varphi_{k_{t} \rightarrow 0}=\frac{K_{2} k_{-1}[\mathrm{~A}]_{s}}{k_{-1}+k_{3}} .
$$

When $K_{2}$ is high, $\varphi$ can be quite large. This leads to the conclusion that the easiest case in which one can distinguish between the models by steady-state results is when $K_{1}$ is small and $K_{2}$ is large. For such a case $\varphi$ is of high value and will not cause any practical problems. However, when both $K_{1}$ and $K_{2}$ are large (with $K_{1} \gg K_{2}$ ), which happens for deamination of amines and dehydration of alcohols (Koubek et al., $1980 \mathrm{a}, \mathrm{b}), \varphi$ is very small and both models yield virtually the same results.

\section{ANALYSIS OF UNSTEADY-STATE CONDITIONS}

\subsection{A stop in the supply of reactant $A$}

It can be assumed that when there are difficulties in discriminating between models under steady-state conditions, some useful results can be obtained based on unsteady state behaviour.

After a stop of reactant $\mathrm{A}$, both models exhibit an immediate rise in their reaction rates. Under an assumption of equilibrium in the second-stage equations [eqs (2) and (2*)] for both models, the maximum reaction rise can easily be calculated (Thullie and Renken, 1990) and an increment in the reaction rate over the steady state can be defined. For model I, this is given as

$$
\Delta R_{1}=\frac{k_{3} K_{1} K_{2}[\mathrm{~A}]_{s}^{2}}{\left(K_{1}[\mathrm{~A}]_{s}+1\right)\left(K_{2}[\mathrm{~A}]_{s}+1\right)+k_{3} / k_{-1}}
$$

and for model II as

$$
\begin{aligned}
\Delta R_{\mathrm{II}} & =\frac{k_{3} K_{1} K_{2}[\mathrm{~A}]_{s}^{2}}{K_{1}[\mathrm{~A}]_{s}\left(1+K_{2}[\mathrm{~A}]_{s}\right)+\left(k_{3}+k_{-1}\right) / k_{-1}} \\
& =\frac{k_{3} K_{1}[\mathrm{~A}]_{s}^{2}}{K_{m}+[\mathrm{A}]_{s}+K_{2}[\mathrm{~A}]_{s}^{2}}
\end{aligned}
$$

For the two models with different sets of kinetic parameters, $\Delta R_{\mathrm{IV}}^{\prime} / \Delta R_{\mathrm{I}}$, after the use of eq. (6), gives

$$
\frac{\Delta R_{\mathrm{II}}^{\prime}}{\Delta R_{\mathrm{I}}}=\frac{k_{3}^{\prime} K_{1}^{\prime} K_{2}^{\prime} H_{1}}{k_{3} K_{1} K_{2} H_{\mathrm{II}}^{\prime}}=\frac{K_{2}^{\prime}}{K_{2}}
$$

So, the reaction rise after a stop for different modcls is not the same for distinct sets of parameters and is a valuable tool in model discrimination. Exactly the same is true for the first case. The difference between the models depends on the ratio of the equilibrium constants of the second reaction.

A very different result is obtained for the third case. A ratio of rate increments gives

$$
\begin{aligned}
\frac{\Delta R_{11}}{\Delta R_{\mathrm{I}}} & =\frac{\left(K_{1}[\mathrm{~A}]_{s}+1\right)\left(K_{2}[\mathrm{~A}]_{s}+1\right)+k_{3} / k_{-1}}{K_{1}[\mathrm{~A}]_{s}\left(1+K_{2}[\mathrm{~A}]_{s}\right)+\left(k_{3}+k_{-1}\right) / k_{-1}} \\
& =\frac{H_{1}}{H_{\mathrm{II}}}
\end{aligned}
$$

which is identical to eq. (7). The result is

$$
\frac{\Delta R_{\mathrm{II}}}{\Delta R_{\mathrm{I}}}=\frac{R_{\text {SII }}}{R_{S I}}
$$

The equality (17) simply says that when reaction rates during steady-state operations are difficult to differentiate, increases in the reaction rate after a stop in the supply of reactant $A$ will be the same. It means that a simple unsteady-state experiment is not going to offer any useful help in model discrimination.

\subsection{Forced concentration oscillations (FCO)}

The mean reaction rate under $\mathrm{FCO}$ between zero and concentration [A] with a very short cycling time can be expressed (Thullie and Renken, 1991) as fol- 
lows:

Model I:

$$
R_{\text {RSSI }}=R_{S I}\left[(1-\gamma)+Q \frac{E(1-\gamma) \gamma}{E(1-\gamma)+F}\right]
$$

Model II:

$$
\boldsymbol{R}_{\mathrm{RSSII}}=\boldsymbol{R}_{S \mathrm{II}}\left[(1-\gamma)+G \frac{D(1-\gamma) \gamma}{D(1-\gamma)+F}\right] .
$$

For each model and several different parameter sets (case 1) it is clear that under FCO one should get different results. However, how different they will be depends on the particular case investigated. The same must hold for two models with different sets of kinetic parameters. Therefore, we can restrict our attention to the third case, which is not recognizable during the stop-effect.

Taking the same $\gamma$ and evaluating the ratio of (18) and (19) (under the assumption $R_{S I} \approx R_{S I I}$ ) gives

$$
\frac{R_{\mathrm{RSSI}}}{R_{\mathrm{RSSI}}}=\frac{1+\frac{G D \gamma}{D(1-\gamma)+F}}{1+\frac{Q E \gamma}{E(1-\gamma)+F}} .
$$

When this ratio is near 1 , the models are difficult to distinguish in this case as well. However, such a situation is not likely to arise since $E \neq D$ and $G \neq Q$. Nevertheless, small $\gamma$ and [A] values should be avoided. For $\gamma=0$ we have a steady state. The ratio (20) may be quite large, even in the case when $\boldsymbol{R}_{\mathrm{RSSI}}$ and $\boldsymbol{R}_{\mathrm{RSSII}}$ are very small and hence hardly measureable.

To discriminate between the models in real systems, it is more convenient to express the difference between the mean reaction rates under $\mathrm{FCO}$ as

$$
M=R_{\mathrm{II}}-R_{\mathrm{I}}
$$

where

$$
\begin{aligned}
R_{\mathrm{I}}= & \frac{k_{3} B}{(E+F) \tau}\left[\frac{E}{F(E+F)}+\frac{C}{(E+F)}\right] \\
& \times \frac{\left(1-P^{-1} U T^{-1} S\right)\left(1-S^{-1}\right)}{\left(1-P^{-1} U T^{-1}\right)}+R_{S I}(1-\gamma) \\
R_{\mathrm{II}}= & \frac{k_{3}}{(D+F) \tau}\left[\frac{D}{F}-\frac{B}{(D+F)}\right] \\
& \times \frac{\left(1-W^{-1} V T^{-1} S\right)}{1-W^{-1} V T^{-1}}+R_{S I I}(1-\gamma) .
\end{aligned}
$$

Equality (21) is written for a finite cycling time $\tau$. First, let us analyse this expression for $\tau=0$ to have some idea about its dependence upon [A] and $\gamma$.

After introducing a mean concentration of $A$ during cycling time $[\overline{\mathbf{A}}]=(1-\gamma)[\mathrm{A}]$, a comparison of both models is shown in Fig. 1. For the sake of comparison, a steady-state reaction rate is also shown. This is denoted by a dashed line. The difference between unsteady-state mean reaction rates $(M)$ is plainly

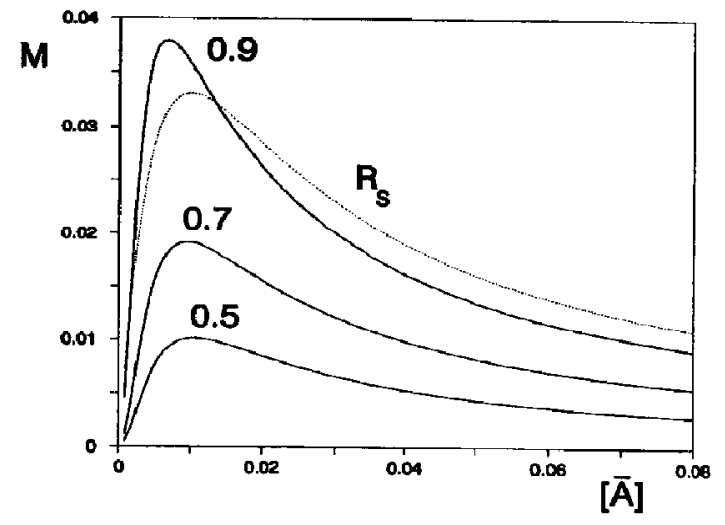

Fig. 1. Mean reaction rate increment as a function of [A] when cycling between zero and [A] with a very small cycling time and for different splits $\gamma=0.5 ; 0.7 ; 0.9$. Input data: $k_{1}=10 ; k_{-1}=0.001 ; K_{2}=100 ; k_{3}=0.1$.

visible. It reaches the highest value at a particular $[\bar{A}]$, so a problem of the optimal choice of $[\bar{A}]$ arises. Figure 1 suggests that the choice $[\bar{A}]=[A]_{\text {sop }}$ may be a good one, especially when $\gamma$ is not close to 1 .

There is no indication how to choose $[\bar{A}]$ for $\gamma$ values near 1 . The best way is to do some experiments for several distinct values of [A]. In any case, quite high values of $[A]$ are anticipated (see Fig. 4; $\tau=0$ ). This is important, because the difference also depends on a split value. The results can be improved by the use of a high split, as indicated by eq. (20). When the split value is decreasing the difference between the models is also decreasing, and at the limit $\gamma=0$ (when a steady state is attained) the models are indistinguishable.

Figure 1 is valid under the condition $\tau \rightarrow 0$. Usually, while performing FCO one should use some small but finite cycling time. When the cycling time increases, the difference between both models decreases (Figs 2 and 3). This is obvious, because then a quasi-steady state is approached. The rate of this decrease is especially high for the [A] values that give the most desirable results (the largest difference between the reaction rates). This fact is confirmed by Figs 2 and 3 . This suggests that during FCO experiments one should make a search for the optimal [A] (which yields the maximum difference) for each particular $\tau$ applied. The indications $\left([\bar{A}]_{\mathrm{og}}=[\mathrm{A}]_{s_{\mathrm{op}}}\right)$ obtained under the assumption $\tau=0$ may be helpful, but not necessarily true for $\tau \neq 0$.

When a cycling time increases, the maximum difference between the models under FCO is shifted in the direction of small [A], as shown in Fig. 4. The dashed curve denotes the case when a cycling time approaches its limit value of zero. The shape of this curve is not the same as in Fig. 1 because it is now given as a function of a concentration [A] during the second part of the cycle. In Fig. 1 it is a function of the mean concentration of $[A]$. This is close to the curve for a cycling time equal to one. It gives the limiting 


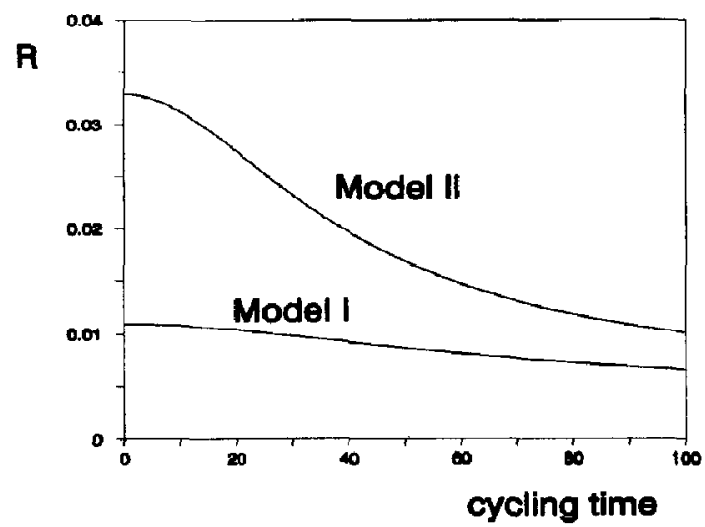

Fig. 2. Comparison of mean reaction rate for model I and model II when cycling between zero and $[A]=0.02$ for different cycling times. Input data: $k_{1}=10 ; k_{-1}=0.001$; $K_{2}=100 ; k_{3}=0.1 . \gamma=0.9$.

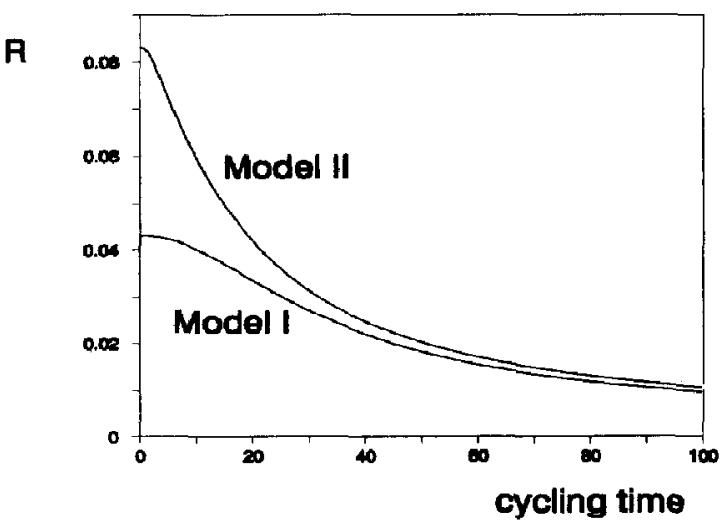

Fig. 3. Comparison of mean reaction rate for model $I$ and model II when cycling between zero and [A] $=0.06$ for different cycling times. Input data: $k_{1}=10 ; k_{-1}=0.001$; $K_{2}=100 ; k_{3}=0.1 . \gamma=0.9$.

upper bound of the optimum of [A] which should be applied when $\tau \rightarrow 0$. This optimum will be more important for long cycling times.

The mean reaction rate increment for several splits is shown as a function of concentration [A] during the second part of the cycle in Fig. 5 for one chosen $\tau=10$. A maximum is obtained for every split value. The highest values of $M$ are obtained for the highest split. A shift of maximum is observed towards smaller $[A]$ values in comparison with the case $\tau=0$.

\section{CONCLUSIONS}

The two basic models proposed in the literature for reactions with a stop-effect are sometimes difficult to distinguish under steady-state conditions. Three cases were investigated in which the same fit to the experiments was produced by

(1) several sets of kinetic parameters for one model,

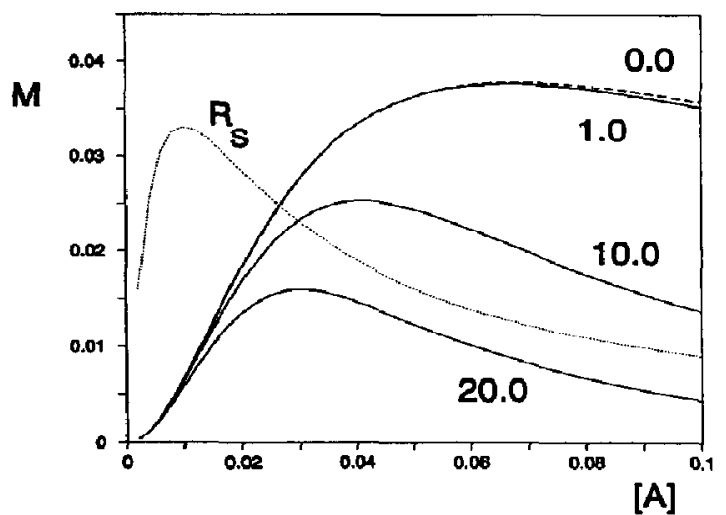

Fig. 4. Mean reaction rate increment as a function of concentration [A] during the second part of the cycle for different splits $(\tau=0 ; 1.0 ; 10 ; 20)$ and with $\gamma=0.9$. Input data: $k_{1}=10 ; k_{-1}=0.001 ; K_{2}=100 ; k_{3}=0.1$.

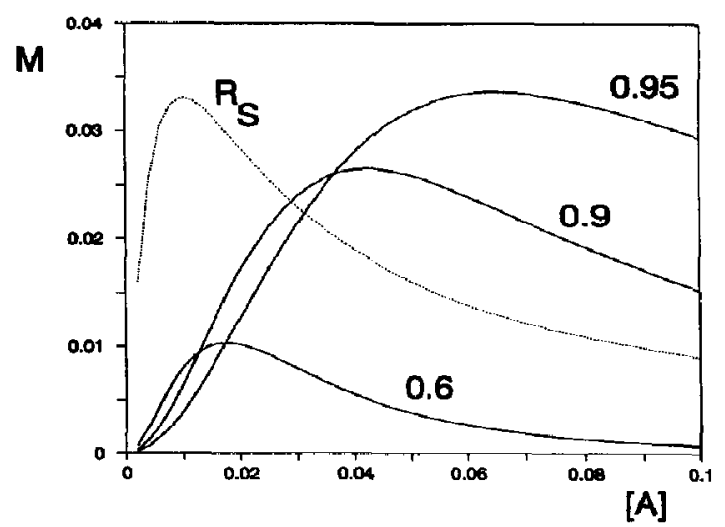

Fig. 5. Mean reaction rate increment as a function of concentration [A] during the second part of the cycle for different splits $(\gamma=0.6$ : 0.9 : 0.95$)$ and with $\tau=10$ Input data: $k_{1}=10 ; k_{-1}=0.001 ; K_{2}=100 ; k_{3}=0.1$.

(2) two models with different sets of kinetic parameters, and

(3) two models with the same set of kinetic parameters.

It is proved in this work that a simple transient behaviour after a stop in the supply of the reactant is a valuable tool only for the first two cases. It means that one can obtain different results for each parameter set. The difference in the reaction rate increments is profound when the equilibrium constants of the second step differ widely.

It was also proved that for the third case the transient behaviour of both models after a stop is identical, so it is useless for model discrimination.

A considerable difference in the mean reaction rate under FCO can be observed for every case listed. It is largest for $\tau \rightarrow 0$ with high split $(\gamma \rightarrow 1)$ and a high concentration of $[\mathrm{A}]$ during the second part of the 
cycle. The difference decreases with an increase of cycling time $\tau$. For every finite $\tau$ there are values of $\gamma$ and [A] which yield a maximum difference.

Generally, it is seen that when the difference between the mean reaction rate under FCO for the models is small, it lasts much longer when the cycling time is increasing, and when it is high its decrease is relatively rapid.

For the third case, the best way to discriminate between the models is that after completing the estimation of kinetic parameters in the standard way for one of the aforementioned models, one should calculate the $\varphi$ value (eq. 11). This gives some recognition whether or not the second model can be applied with the same success. If $\varphi$ is small, say 0.01 , performing FCO is advised to discriminate between them. It is good to start FCO with as small a cycling time as possible. The split value and the concentration [A] should result from numerical maximization of $\boldsymbol{M}=\boldsymbol{f}(\gamma,[\mathrm{A}])$ [eq. (21)]. As a first approximation, high values of $[A]$ and $\gamma$ may be applied.

Sometimes an investigation of the catalyst surface shows which model is closer to reality. Such a situation takes place for the deamination of amines. It is known that in these cases two different kinds of active sites are involved. However, such situations are not always encountered and sometimes a transient experiment may be a valuable tool.

Recently, a new model of a stop-effect has been proposed by Moravek (1992) for low alcohol dehydration on alumina. It is based on the Ipatiev mechanism of bimolecular substitution and may be simplified to the mathematical description given by model II.

Acknowledgement-One of the authors (J. Th.) thanks the State Committee of Scientific Research (KBN) for partial financial support of this project by grant PB 812/3/91.

\section{NOTATION}

[A] dimensionless A concentration

[AS] dimensionless AS concentration

$B$ dimensionless constant $\left(=k_{1}[\mathrm{~A}]\right)$

$C$ dimensionless constant

$\left[=K_{2}[\mathrm{~A}] /\left(K_{2}[\mathrm{~A}]+1\right)\right]$

$C_{0}$ total concentration (the same during a steady state and a transient operation)

$\left[\mathrm{mol} \mathrm{m} \mathrm{m}^{-3}\right]$

$D \quad$ dimensionless constant

$\left[=k_{1}[\mathrm{~A}]\left(K_{2}[\mathrm{~A}]+1\right)\right]$

$E \quad$ dimensionless constant $\left(=k_{1}[\mathrm{~A}]-k_{3} C\right)$

$F \quad$ dimensionless constant $\left(=k_{3}+k_{-1}\right)$

$G \quad$ dimensionless constant

$[=(D+F) / B-F / D]$

$H_{I} \quad$ dimensionless constant

$\left\{=\left(K_{1}[\mathrm{~A}]_{s}+1\right)\left(K_{2}[\mathrm{~A}]_{s}+1\right)+k_{3} / k_{-1}\right\}$

$H_{\mathrm{II}}$ dimensionless constant

$\left\{=K_{1}[\mathrm{~A}]_{s}\left(K_{2}[\mathrm{~A}]_{s}+1\right)\right.$

$\left.+\left(k_{3}+k_{-1}\right) / k_{-1}\right\}$

$K_{1} \quad$ equilibrium constant $\left(=k_{1} / k_{-1}\right)$

$K_{2}$ equilibrium constant $\left(=k_{2} / k_{-2}\right)$ $k_{i}$

dimensionless rate constant $\left(=\tau_{v} \kappa_{i} C_{0}\right.$ for $i=1,2 ;=\tau_{0} \kappa_{i}$ for $i=-1,-2$;

$k_{3}=\kappa_{3}\left(L_{2}^{*}\right) \tau_{0}$ for model I;

$k_{3}=\tau_{0} \kappa_{3}$ for model II).

$\left(L_{2}^{*}\right) \quad$ total concentration of active sites $S_{2}$ $\mathrm{mol} \mathrm{m}^{-2}$

$M \quad$ a difference between mean reaction rates $\left(=\bar{R}_{\text {II }}-\bar{R}_{\mathrm{I}}\right)$

$P \quad$ dimensionless constant $[=\exp (E \tau)]$

$Q \quad$ dimensionless constant

$[=(E+C F) /(E-C E)]$

$R_{S} \quad$ dimensionless steady state reaction rate

$\bar{R} \quad$ dimensionless mean reaction rate during FCO

$S \quad$ dimensionless constant $[=\exp (F \gamma \tau)]$

$T$ dimensionless constant $[=\exp (F \tau)]$

$t$ dimensionless time $\left(=9 / \tau_{0}\right)$

$U$ dimensionless constant $[=\exp (E y \tau)]$

$V \quad$ dimensionless constant $[=\exp (D \gamma \tau)]$

$W \quad$ dimensionless constant $[=\exp (D t)]$

\section{Greek letters}

$\gamma \quad$ cycle split

$\kappa_{i} \quad$ rate constant $\left(\left[\mathrm{m}^{3} \mathrm{~mol}^{-1} \mathrm{~s}^{-1}\right]\right.$ for $i=1,2$; $\left[1 \mathrm{~s}^{-1}\right]$ for $i=-1,-2$ and for $i=3$ model II; $\left[\mathrm{m}^{2} \mathrm{~mol}^{-1} \mathrm{~s}^{-1}\right]$ for $i=3$ model I)

9 time, $\mathbf{s}$

$\tau=\tau_{c} / \tau_{0}$

$\tau_{0} \quad$ space time of the differential reactor, $s$

$\tau_{c} \quad$ cycle time, $s$

\section{Subscripts \\ op optimum \\ $S \quad$ steady state \\ RSS relaxed steady state \\ I model I \\ II model II}

\section{REFERENCES}

Bailey, J. E. and Ollis, D. F., 1977, Biochemical Engineering Fundamentals. McGraw-Hill, New York.

Koubek, J., Pasek, J. and Ruzicka, V., 1980a, Exploitation of a nonstationary kinetic phenomenon for the elucidation of surface processes in a catalytic rcaction, in Proceedings of 7th International Congress on Catalysis, Tokyo, Vol. 7B, p. 853.

Koubek, J., Pasek, J. and Ruzicka, V., 1980b, Stationary and nonstationary deactivation of alumina and zeolites in elimination reactions, in Catalyst Deactivation. Elsevier, Amsterdam.

Moravek, V., 1992, Steady-state and transient kinetics of displacement adsorption and educt inhibition in dehydration of alcohols on alumina, in Progress in Catalysis. Elsevier, Amsterdam.

Nowobilski, P. J. and Takoudis, Ch. G., 1986, Periodic operation of chemical reactor systems: are global improvements attainable? Chem. Engng Commun. 40, 249.

Thullie, J. and Renken, A., 1990, Transient behaviour of catalytic reactions with stop-effect. Chem. Engng Commun. 96, 193.

Thullie, J. and Renken, A., 1991, Forced concentration oscillations for catalytic reactions with stop-effect. Chem. Engng Sci. 46, 1083. 